\title{
Recherche d'une Loi périodique sur l'hydraulicité (I)
}

\section{Do rainfall variations obey a periodic law}

\author{
PAR H. CHAMLYYOU \\ CheF de Senvace de L'Enenge Electroue de SA S.N.C.F. \\ English synopsis, p. 527.
}

La succession des années siches que nous venons de subir a de nouveau excité l'intérêt que présenterait la connaissance des périodicités météorologiques.

Après la libération, la pénurie d'énergie s'est présentée comme le problème $\mathrm{n}^{\circ} 1$ à résoudre pour la remise en route de notre pays et de l'Europe. Le charbon manquait, il fallait des devises et des bateaux pour le faire venir d'Amérique. Nous disposions chez nous de réserves hydrauliques naturelles encore inexploitées. Les responsables de l'économie nationale se sont donc tout naturellement orientés vers l'équipement des chutes et la construction de barrages pour régulariser les débits naturels des cours d'eau, naturellement irréguliers.

On postulait ainsi implicitement qu'il y aurait dans nos rivières et derrière nos barrages une certaine quantité d'eau, donnée par des relevés. Malheureusement, ces relevés n'ont une précision suffisante que depuis un demi-siècle environ. Ies moyennes ont été mises en défaut par la succession d'une série d'années sèches et l'on s'est demandé si les milliards qui avaient été investis dans les chutes et les barrages, et dont malheureusement nous ne possédons qu'une quantité cruellement limitée, avaient été employés au mieux. On a douté des statistiques, et mème on est allé jusqu'à se demander si cette pénurie ne correspondait pas à une variation durable dans le climat de notre pays comme celle qui a créé le Sahara. Dans ces conditions, d'ici quelques an- nées, les barrages derrière lesquels il y aurait moins d'eau perdraient de leur intérêt.

Par ailleurs, la pénurie de charbon s'est résorbée, grâce notamment aux progrès faits pour utiliser les produits de basse qualité. Certains prévoient même qu'il y aura dans peu de temps des excédents risquant de rester inemployés, et les programmes d'équipement qui sont sensibles non seulement à des considérations techniques mais encore à des influences sentimentales, presque de mode, ont évolué. On a redécouvert les vertus des centrales thermiques qui coûtent moins cher que les centrales hydrauliques, sont réalisables dans un délai plus court et ne sont pas soumises aux fantaisies de la métcorologie.

Si, au contraire, il existe une variation périodique de l'hydraulicité, et si cette périodicité était connue, on serait rassuré quant à l'utilité future des équipements en cours ou encore en projet.

Ce n'est d'ailleurs pas seulement en matière d'énergie que la connaissance de celte périodicité serait importante. Elle s'appliquerait en effet a tous les phénomènes météorologiques climatiques : température, pression, électricité atmosphérique. Elle commanderait, comme au temps de Joseph, les cycles d'abondance agricole ou de pénurie des récoltes, done de vie facile ou d'existence difficile - peut-être de famine, en certains pays comme la Chine et l'Inde. Les troubles sociaux étant liés aux conditions matérielles de vie, les gouvernants ont d'autant plus de difficultés avec leurs administrés que ceux-ci sont plus 
malheureux. C'est donc l'ordre politique et social qui, à son tour, serait influencé par ces cycles météorologiques.

$$
\text { 事 }
$$

Les recherchess faites dans cette voie sont nombreuses et anciennes. Depuis Joseph, déjà cité, qui avait prédit la période de sept ans, on ne sait trop d'après quelles données, de nombreases périodes ont été proposées. Je ne citerai parmi elles que la loi de Brïckner - de 35 ans - valable pour l'Europe occidentale, la plus connue; et celle d'Arago, de 744 ans.

Plus récemment, à l'occasion diune enquête effectuée en 1936-1937 par la revue la Météorologie sur les périodicités en météorologie - enquête à laquelle ont participé plusieurs spécialistes francais et itrangers, et dont les conclusions ont d'ailleurs été dans l'ensemble plutôt négatives - l'abbé Gabrirc parle d'une période de $\$ 72$ ans (exactement la moitié de celle d'Arago), correspondant à des époques où te soleil, la terre et la lune se retrouvent tris approximativement dans les mèmes situations respectives.

La difficulté d'élablissement d'une telle loi provient de labsence de données précises sur une période sulfisamment longue. Aussi a-t-on essayé do pallier cette pénurie de documents an rattachant les phónomènes météorologiques à des phénomènes sur lesquels on possède des renseisnements plus lointains, ces derniers estimés en corrélation plus ou moins étroite entre les premiers.

L'une de ces corvélations fut signalée par W. Hercher. Celui ci releva le prix du blé, qui dépend évidemment de l'abondance ou de la pénurie des récoltes, done des conditions météorologiques, dans its archives des Chambres de Commerse entre 1650 et 1713 , e'est-ia-dire pendant une période de 63 ans. Il constata que la végétation était en déficit durant les années où le soleil avait peu de taches et se révélait au contraire plus abondante quand les taches étaient plus nombreuses à la surface de l'astre.

En 1872, le docteur Ménrum, directeur de l'Observatoire de l’ìle Maurice, étudia la périodicité des cyclônes particulièrement violents dans cette région de l'ocćan Indien. Son altention se porta sur les conséquences de ces terribles tempêtes, les naufrages de navires qui les accompagnent, el il constata que le nombre des épaves qui viennent échouer sur les côtes de l'île Maurice suivait aussi les mêmes variations que celles res taches du soleil.

Deux ans plus tard, en 1874, Norman LucBEYER découvrait que le cycle de pluies de lî̀le de Ceylan avait une périodicité égale à celle des taches solaires.

Celte correspondance fut encore retrouvée avec les pluies dans d'autres régions : Inde, Cap de Bonne Espérance, Ecosse, avee les grandes famines de l'Inde et mème avec les années de vin de haute qualité dans le Bordelais.

La relation de cause à effet provient du fait que la radiation solaire est la plus élevée aux ćpoques qui présentent le maximum de taches ot cue cette augmentation de température se traduit par une augmentation de température à la surface de la terre, accompagnée des variations de pression atmosphéricque elles-mêmes en relation avec les rents et leur intensite. Or, la dimension des taches solaires varie périodiquement suivant un cycle d'une durée de 11 ans.

Par: la suile, on pensa que les taches solaires n'étaient pas seciles en jeu et que les protub:rances solaires, ces formidables éruptions qui élèvent des gerbes de flammes de quelques centaines de mille de $\mathrm{km}$, au-dessus de la surface du soleil, sur des surfaces pouvant atteindre le $1 / 10$ de la surface du soleil, devaient avoir une influence considérable sur les radiations solaires.

Ces protubérances se manifestent également périodiquement, suivant un cycle de 3 ans $\mathrm{ct}$ $7 / 10$.

On découvit enfin qu'il y avait correspondance entre les variations des taches solaires et les perturbations du magnétisme terrestre.

Malheureusement, les nombreux relevés de débits faits sur nos rivières n'ont pas confirmé l'existence de ces périodes auxquelles on avait songé à priori.

L'opinion généralement admise aujourd'hui est que l'action des taches solaires, si elle existe, est très faible. D'une manière plus générale, les spécialistes sont à peu près d'accord pour reconnaître qu'aucune des nombreuses périodicités proposées n'a reçu des faits aucune confirmation satisfaisante.

Peut-il exister une loi de périodicité permettant de prévoir les années sèches et les années hamides?

Dans la nature, de nombreux phénomenes prísentent des alternances d'intensité qui se succèdent avec une grande régularité.

Il y a d'abord les phénomènes varament périodiques dans lesquels il existe une ou plusicurs variables dont les dérivées secondes ont comme termes prépondérants ou bien des quantités proportionnelles aux variables elles-mêmes et de signe contraire ou bien une quantité indépendante et périodique (oscillations propres et oscillations forcées). La période se trouve inscrite dans l'équation différentielle. 
Il y a en outre de nombreux phémomènes de relaxation :

- battements du cœur; broutement des outils de machines.

re modile le plus simple, et dont l'explication est la plus élémentaire, est celui des appareils d. chasse automatiques. Dans ces phénomenes, le déclenchement d'une alternance est subordonnéc à une cause extérieure et de faible importance relative.

On pourrait penser que le débit d'une rivière ou sa cause se ratlache à un modele de relaxation. Nous examinerons ultérieurement cette possibilité; mais pour essayer de mettre en évidence ces alternances régulières, nous avons postulé que la périodicité, si elle existe, devait provenir de phénomènes eux-mêmes périodiques.

Or, les seuls de ce genre que nous connaissions sont les phénomènes astronomiques, et ceux-ei ont par ailleur's une influence certaine sur notre planc̀te.

-_ Le soleil d'abord a une action prépondírante : il fait succeder le jour et la nuit, puis les saisons, avec toutes leurs conséquences calorifiques, barométriques et hydrologiques. Sur certaines régions de la terre, cette influence est d'une netteté particuliere puisqu'clle est à l'origine des moussons et des vents alizés.

Le cycle correspondant est le cycle évident de lannée.

- Si le soleil intervient par sa masse, sa ten1pérature et ses radiations, la lune intervient par sa distance de la terre beaucoup plus faible. C'est elle qui est l'élément capital des marées. Son action sur les masses gazeuses de l'atmosphìre est evidemment plus faible que sur les masses d'eau des océans puisque les densités de ces masses de vapeur et d'air sont mille fois plus faibles que celle de l'eau. Cette action est écrasée par l'influence calorifique du soleil beaucoup plus inportante, mais elle n'est sans doute pas négligeable. Il existe des marées atmosphériques, comme des marées océaniques. L'influence lunaire sur les vents alizés paraìt également bien démontrée.

Au cycle lunaire pur de 28 jours s'ajoute d'ailleurs le cycle de 19 ans au bout duquel la lune se retrouve dans la mème situation par rapport à la terre. Ce cycle est à l'origine du nombre d'or, que la religion a utilisć.

- Les autres planètes et les étoiles ont également incontestablement une influence, mais infiniment plus faible. Pourtant on peut noter que la baisse de température constatée aux environs du 12 mai (saints de glace) et le net réchauffement qui se produit exactement six mois plus tard, autour du 11 novembre (été de la Saint-
Martin) ont été expliqués par la rencontre de la terre avec l'orbite ellipticue d'essaims d'astérö̈des qui circulent autour du soleil.

- Il ne parait done pas aventureux de conclure qu'il doit exister des périodes supérieures à celles de l'année en rapport avec les cycles du solcil, de la lune et des autres astres.

Mais ce qui explique partiellement l'échee des recherches qui ont jusqu'ici été tentées, c'est que les irrógularités au cours des périodes principales lunaires et solaires, c'est-à-dire mois et année, provenant de causes multiples terrestres, son! d'un ordre de grandeur nettement supérieur à linfluence cyclique astronomique, résultant des positions relatives du soleil, de la lune, des planètes el des éloiles el étouffent celles-ci.

En bref, lanalyse harmonique de la période principale de lannéc ne décéle pas les harmoniques dordre supérieur parce que les irrégularile's accidentelles sont plus importantes que les variations r'ćsultant de ces harmoniques supéricures.

Si l'on veut mettre en évidence ces derniers harmoniques, il faudrait se libérer de la période principale.

Pour cela, on a pensé au Service de l'Energie Electrique de la S.N.C.F., à prendre comme paramètre les débits moyens relevés à la fin de chaque mois pour la période comprenant le mois considéré et Ias 11 mois précédents; on oblient ainsi wne courbe donmant 1 point par mois, chaque point etant te débit global de 12 mois, c'est-à-dire comprenant les débits d'automme, d'hiver, de printemps et d'été.

Le fait d'avoir un point par mois élimine également l'influence lunaire principale.

Cette méthode a été appliquée aux débits de la Dordogne, à Marèges, dont les valeurs journalières sont comnues depuis 1918 et les valeurs moyennes mensuelles depuis juillet 1903; elle a donné le résultat concrétisé par le graphique stivant (fig. 1):

Cette représentation met en évidence 11 alternances dont la durée varie de 3 à 4 ans, périodicité extrêmement nette à l'exception de l'intervalle de 7 ans allant de 1912 à 1919 pendant laquelle d'ailleurs nous ne sommes pas absolument certain des statistiques.

En outre, si l'on prend la moyenne de chacune de ces alternances élémentaires, on s'apercoil que, partant d'un chiffre faible, cette moyenne s’éleve régulièrement pendant 3 alternances élémentaires, reste sensiblement constante pendant 6 autres, pour s'abaisser ensuite pendant une nouvelle série de 3 .

L'alternance moyenne de 4 ans parait donc se superposer à une période d'amplitude beaucoup plus grande d'au moins une cincuantaine 


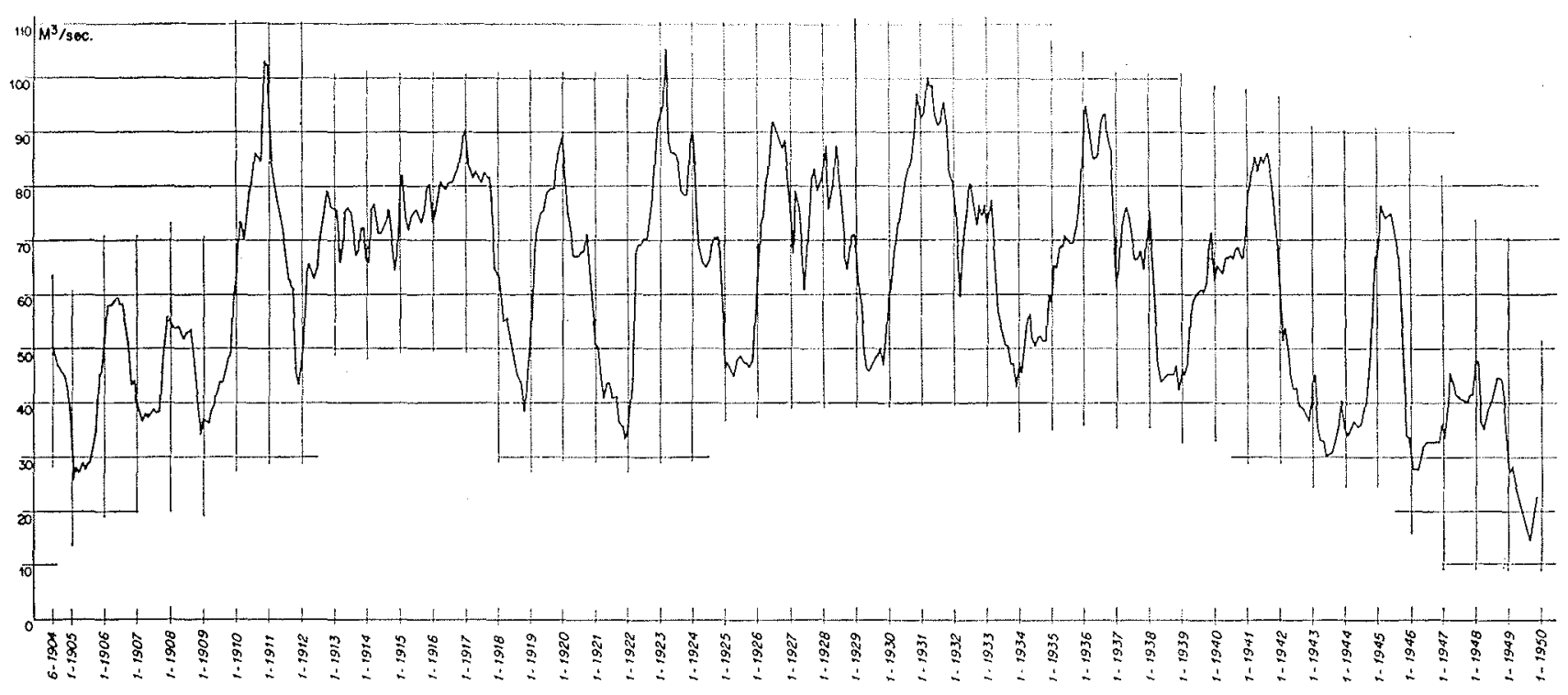

Frg. 1. - La Dordogne a Maríges.

Courbe du débit moyen, en $\mathrm{m} 3 / \mathrm{sec}$, pendant douze mois consécutifs. (Le débit de $50 \mathrm{~m} 3 / \mathrm{sec}$, indiqué en regard de juin 1904 , est le débit moyen observé pendant les douze mois de juillet 1903 à juin 1904 inclus. Le débit de $48 \mathrm{~m} 3 / \mathrm{sec}$, indiqué en regard de juillet 1904 , est le débit moyen observé pendant les douze mois d'aont 1903 à juillet 1904 inclus, et ainsi de suite...)

d'années. Malheureusement, les relevés dont nous disposons s'étendent sur une durée trop courte pour que l'on puisse en tirer une conclusion valable sur l'amplitude de cette période.

Nous avons cherché, vainement d'ailleurs, ì vérifier si une telle périodicité se retrouvail dans d'autres phénomènes, par exemple la croissance. des arbres en Corrèze, comme on l'a déjà fait en rattachant la végétation des sapins de Norvège à la température du Gulf Stream.

Quoi qu'il en soit, si cette périodicité est contestable, il n'en est pas de même du tout des alternances dont le nombre est suffisamment grand pour que leur retour régulier ne puisse être attribué au seul effet du hasard.

Sans doute leurs durées sont-elles différentes les unes des autres, mais cette irrégularité est très explicable.

En effet, le débit des rivières est évidemment fonction des précipitations atmosphériques, ellesmêmes sous la dépendance de la pression et la temperature.

Nous avons cherché la corrélation existant entre les pluies e! les débits. Malheureusement, nous ne disposions pas, pour le bassin versant de la Dordogne à Marèges (bassin de plus de $2.500 \mathrm{~km}^{2}$ de superficie), d'éléments statisticques suffisants, dans le temps et dans l'espace, concernant les pluies. Notre étude de corrélation entre les pluies et les débits n'a donc pas donné de résultats salisfaisants.
Nous avons pu néanmoins à cette occasion faire les observations suivantes :

Le Massif Central est à régime presque entièrement pluvial et le phénomène de rétention et de régularisation dû aux neiges et aux glaciers intervient relativement peu.

Au contraire, la régularisation par laction dí sol et de sa végétation est importante. Nous avons étudić cet effet, qui est extrèmement utile pour les prévisions des débits à court terme et même à moyen terme.

Les conclusions auxquelles nous sommes parvenus sont les suivantes :

Tout se passe comme si l'ensemble du bassin versant constituait, du fait de la nature du sol, de la végétation, etc., un réservoir fictif ou plutòt un ensemble de réservoirs fictifs correspondant chacun à une zone déterminée du bassin rersant.

Nous avons pu estimer la capacité de ce réservoir fictif à environ 120 millions de $\mathrm{m}^{3}$. Ce réservoir fictif, que l'on peut encore comparer à une éponge, intervient de la facon suivante :

En période de pluie, une première partie de leau qui tombe ruisselle, une deuxième partie est retenue dans ce réservoir fictif jusqu'à satulration. Après cessation de la pluie, cette éponge, ou ce réservoir fictif, restitue l'eau, les débits diminuent au fur et à mesure que le volume en réserve est lui-mème plus petit, pour tendre pratiquement rors 0 , si la sécheresse persiste trop longtemps. 
Les courbes types de remplissage, comme celles de vidange, peuvent se déterminer en fonction de divers parametres. Nous les donnerons dans une autre communication.

A défaut de correspondance entre les débits et les pluies, nous avons cherché une correspondance entre les dćbits et les pressions, les variations de ces dernières embrassant des zones beaucoup plus étendues que celles des chutes de pluie et permettant ainsi d'utiliser des renseignements barométriques même extérieurs au bassin versant. Si l'on regarde le graphique de la figure?, on constate qu'effectivement il y a une correbation très étroite entre les pressions et les débits,
Quoiqu'elle ait été établie pour une portion très réduite de notre territoire, il serait intéressant d'appliquer la méthode proposée à d'autres régions et à des cours d'eau d'autres massifs, les Alpes et les Pyrénées notamment, pour vérifiel si cette loi a un caractère général.

Il nous apparait toutefois que, pour l'objet qui nous occupe, la Dordogne est un témoin fidèle, parce que le Massif Central, ne possédant pas de glaciers, n’a pas les reports interannuels qui déforment la loi principale, comme ce serait probablement le cas pour le Rhin (1) et le Rhone. Les variations des glaciers alimentant ces fleuves compensent en effet dans une certaine mesure,

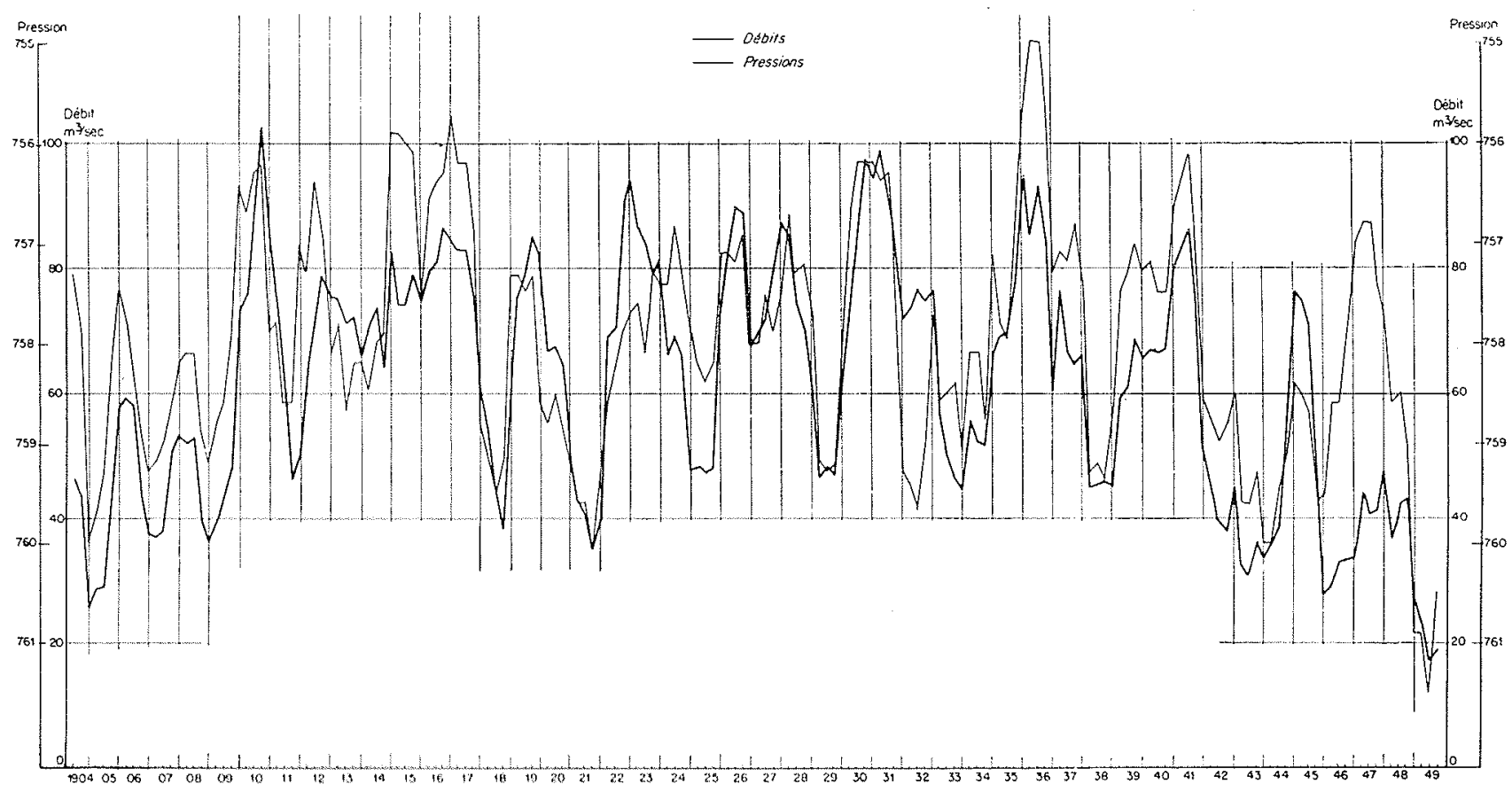

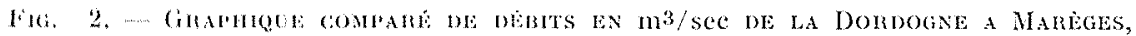

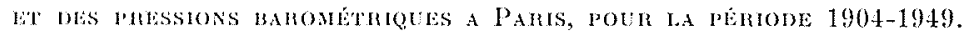

(Les valeurs de debit et de pression correspondant à un trimestre de réference, sont les valenrs moyennes de ces deux variables pendant les quatre trimestres consécutif's comprenant te trimestre consideré et les trois précédents.)

et peut-être encore plus étroits entre la variation des débits el la variation des pressions. Une telle correlation semblerait appuyer lhypothèse que les alternances constituent un phénomène de relaxation plutôt qu'un phénomène de périodicilé pure.

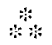

Quoi qu'il en soit, il nous a paru intéressant de faire connaitre la loi des alternances dans le débit de la Dordogne, loi d'autant plus intéressante que de nombreux échees avaient été enregistrés jusqu'ici pour mettre en évidence une telle loi. par les apports provenant de leur propre substance, la faiblesse des débits des années sèches et chaudes.

En contrepartie, il faut remarquer que la po-

(1) Dans une étude du Dr Orsterhaus, publiée en 1946 par le Service Fédéral des Eaux de Berne et relative aux variations du débit du Rhin à Bàle cntre 1908 et 1944, l'auteur fait neammoins ressortir également une alternance r'égulière d'environ quatre ans pour la période postérieure à 1917. Par contre, pour la période antérieure da 1917, les alternanees sont moins régulières. Il serait peutêtre intéressant de reprendre l'étude du Rhin en appliquant la méthode que nous avons suivie pour la Dordogne. 
sition de la France n'est pas des plus avantageuses pour celte recherche. La position de l'océan Indien entre les énormes masses terrestres de l'Asie centrale et de l'Afrique donne à ha mousson une régularité que nous ne retrouvons pas dans nos vents d'ouest. La position de l'Atlantique et de notre Europe ćlant beaucoup moins favorable, toutes les influences perturbatrices arrivent à masquer la périodicité d'un phénomène qui devrait à priori se manifester aussi régulièrement que dans l'Inde.

Il est done probable que ce sont des relevés faits autour de l'océan Indien qui permettraient de mettre en évidence le plus aisément les périodicités d'ordre supéricur à colles des cycles lunaires et solaires, si elles existent.

En bref, que ces alternances soient d'origine de relaxation ou bien qu'elles soient une perio- dicité pure, la cause du phénomène : mécanique ou astronomique, reste a expliquer. Mais pour les années les plus prochaines, il semble que nous puissions conclure que nous entrons dans une période de quatre ans pendant laquelle l'hydraulicité sera plus devée qu'en 1949. Cette hydraulicité doit être plus grande encore en 1951 qu'en 1950, puis elle serait suivie d'une déeroissance jusqu'en 1953.

La moyenne générale de cette alternance scra-l-elle supélieure ou inférieure à celle des quatre dernières années?

- Les relevés ci-dessus ne permettent pas de conclure, mais nous espérons que la méthode appliquée à des phínomines météorologiques en corrélation avec les débits des rivières et connus quantitativement, depuis un plus grand nombre d'années, permetront de lever cette incertitude.

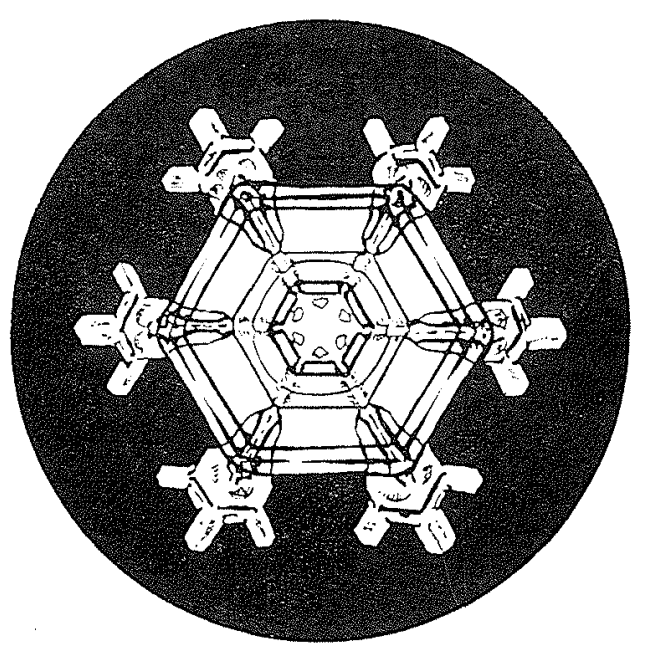

